\title{
Antenatal Ovarian Torsion Presenting With Features of Intestinal Obstruction in a Neonate
}

\section{Jayateertha Joshi ${ }^{1}$, Nutan Kamath ${ }^{2}$, Jyoti Ramnath Kini ${ }^{3}$, Jayashree K$^{2}$, Suchetha $\mathbf{R a o}^{2}$ and Sowmini P Kamath ${ }^{2}$}

${ }^{1}$ Department of Paediatric Surgery, Kasturba Medical College, Mangalore, Manipal Academy of Higher Education, Manipal, Karnataka, India

${ }^{2}$ Department of Paediatrics, Kasturba Medical College, Mangalore, Manipal Academy of Higher Education, Manipal, Karnataka, India

${ }^{3}$ Department of Pathology, Kasturba Medical College, Mangalore, Manipal Academy of Higher Education, Manipal, Karnataka, India

\section{Correspondence:}

Jayateertha Joshi

Department of Paediatric Surgery,

Kasturba Medical College,

Mangalore,

Manipal Academy of Higher Education,

Manipal,

Karnataka, India

E-mail: jaya.joshi@manipal.edu

DOI: $10.3126 /$ jnps.v40i3.28788

Submitted on: $2020-05-06$

Accepted on: 2020-06-24

Acknowledgements: None

Funding: Nil

Conflict of Interest: None declared

Permission from IRB: Yes

To cite this article: Joshi J, Kamath N, Kini $J R$, Jayashree $\mathrm{K}$ and Rao S. Antenatal Ovarian Torsion Presenting With Features Of Intestinal Obstruction In A Neonate. J Nepal Paediatr Soc. 2020;40(3):265-9.

\section{ABSTRACT}

With the advent of antenatal ultrasonography (USG), detection of neonatal intra-abdominal cystic lesions is on the rise. We present a neonate who was detected antenatally to have a cystic lesion per abdomen and presented with features of intestinal obstruction on first postnatal day. Emergency exploratory laparotomy revealed a left torsed ovarian cyst compressing the ileoceacal junction. Left oophorectomy was done and ovarian torsion was confirmed by histopathology examination. The USG abdomen at six months follow up showed a well visualised right ovary.

Key words: laparotomy; newborn; ovarian cysts; ultrasonography

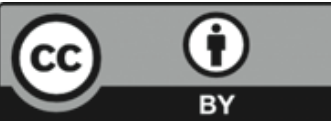

This work is licensed under creative common attribution 3.0 license 


\section{INTRODUCTION}

Ovarian cysts are commonly detected in female foetuses with an estimated incidence of around one in 2600 pregnancies. ${ }^{1}$ Often ovarian cysts are detected antenatally towards the end of second and beginning of third trimester because of extensive usage of ultrasonography. The majority of cysts are small simple cysts that resolve spontaneously. Larger cysts usually are non-resolving and are associated with complications, such as ovarian torsion, haemorrhage within cyst, birth dystocia, mass effect on nearby structures and auto amputation. ${ }^{2,3}$

\section{CASE REPORT}

A term AGA female neonate was born to a $\mathrm{G}_{2} \mathrm{P}_{1} \mathrm{~L}_{1}$ mother with no maternal risk factors by Caesarean section with the indication of previous Caeserean section. Antenatal USG detected a cystic lesion (5.1 $\mathrm{cm} \times 2.4 \mathrm{~cm})$ in the abdomen of the baby at the end of second trimester. Baby had passed meconium and urine within 24 hours of birth. Baby presented with feeding intolerance, bilious vomiting, abdominal distension, and features suggestive of intestinal obstruction on first postpartum day. On clinical examination a mobile cystic mass was palpable in the periumbilical and right lumbar region. USG abdomen detected two well-defined cystic lesions measuring $4.8 \mathrm{~cm} \times 2.7 \mathrm{~cm}$ and 5.5 $\mathrm{cm} \times 2.6 \mathrm{~cm}$ in the periumbilical region and sub
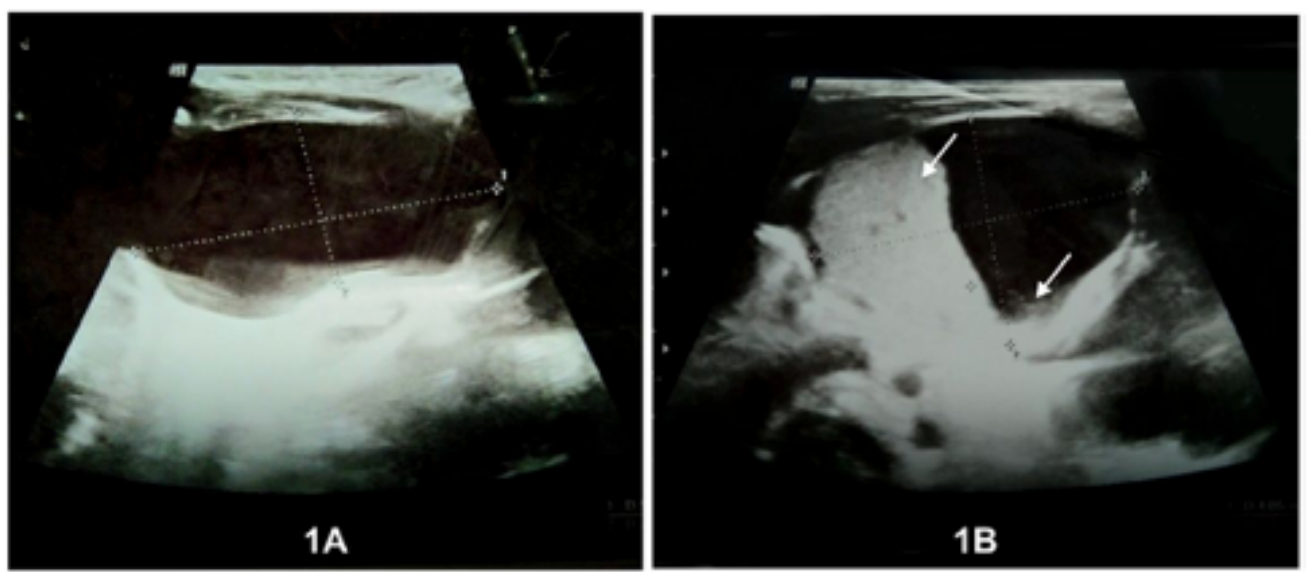

Figure 1A: Postnatal ultrasonography of cyst in the periumbilical region

Figure 1B: upper arrow showing debris and lower arrow showing fluid level within the cyst hepatic region. (figure 1A,1B). One of the cysts showed fluid debris levels. (figure 1B)

When a female foetus is diagnosed to have cystic abdominal mass, differential diagnoses such as ovarian cysts, urachal cysts, cystic teratoma, intestinal duplication anomalies, mesenteric cysts, and intestinal obstruction should be considered. In our case, the neonate presented with features of intestinal obstruction and ultrasonography showed evidence of two cysts in the subhepatic region and periumblical region, with one cyst having fluid debris levels, thus narrowing our diagnosis to duplication, mesenteric or ovarian cysts. MRI that was planned could not be done as the baby had worsening of symptoms requiring an immediate exploration.

A right lumbar transverse incision was done and exploratory laparotomy revealed a dark brown coloured cystic lesion arising from the left ovary. The cystic lesion was measuring $5 \mathrm{~cm} \times 6 \mathrm{~cm}$ with hemorrhagic fluid in it and cyst couldn't be separated from the left ovary (figure 2). This mass was compressing the ileocecal junction with proximal dilated bowel loops. Bowel inspected was normal. Right side ovary, both fallopian tubes and uterus were normal. Left oophorectomy was done.

Gross examination of the excised cyst, (figure 3A) showed a dark brown uni-locular cyst measuring 7 $\mathrm{cm} \times 6 \mathrm{~cm} \times 4.5 \mathrm{~cm}$ containing hemorrhagic fluid 


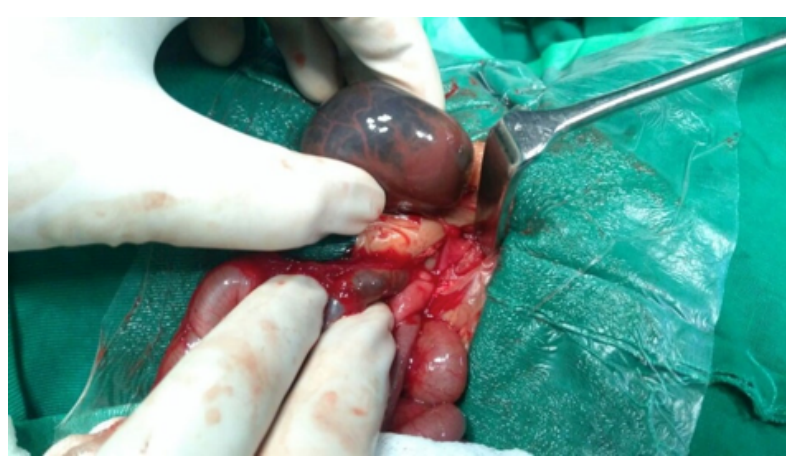

Figure 2. Cyst with ovary showing torsion at pedicle

along with peripheral focal solid ovarian tissue. On histopathologic examination of the cyst, multiple sections were studied (figure 3B, 3C) which showed a cyst wall with hemorrhagic necrosis with partly viable ovarian stroma. (figure $3 \mathrm{D}, 3 \mathrm{E}$ )
Supportive care was given at the neonatal intensive care unit. On second post-operative day, baby was started on breast feeds and discharged on day six of post-natal life. At six months follow up, ultrasound of the abdomen showed a well visualised right ovary with baby's growth within centiles.

\section{DISCUSSION}

Though ovarian cysts are common; its genesis remains controversial. Exposure of the foetus to foetal and maternal gonadotrophins, maternal conditions with increased levels of beta-HCG such as gestational diabetes, toxemia of pregnancy and $\mathrm{Rh}$ iso-immunisation are implicated. ${ }^{2}$

Neonatal ovarian cysts as per Nussbaum's classification are classified as simple/ uncomplicated and complex / complicated cyst. $^{3}$
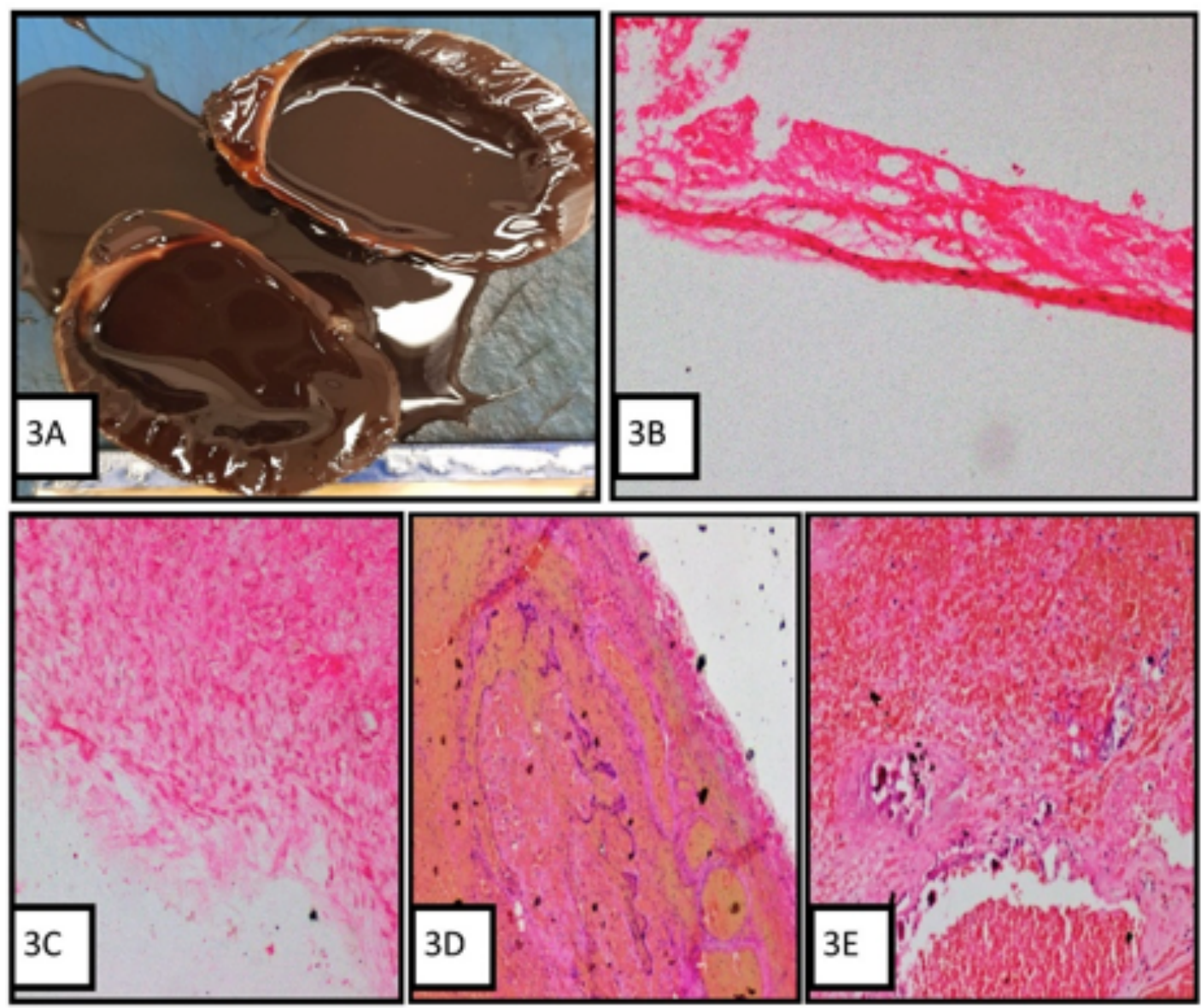

Figure 3A: Gross specimen of left ovarian cyst with torsion

Figure 3B: Cyst wall with attenuated lining epithelium

Figure 3C: Cyst wall with residual ovarian stroma

Figure 3D. 3E: Areas of hemorrhagic necrosis and foci of calcification 
This classification is based on ultrasonography patterns of the cysts with histopathology findings. Simple cysts are usually unilateral, unilocular, homogeneous, anechoic, and thin walled. Complex cysts contain blood clots, fluid debris, septations, solid structures and are usually thick walled. ${ }^{3}$ The presence of fluid debris appears to be a specific sign of cyst torsion ${ }^{3}$ and was present in one of the cysts in our case.

Because of the small size of pelvis in neonates, and in the presence of a long pedicle, the cyst from ovaries may occupy the mid / upper abdomen or even the contralateral side, with torsion of the ovary being the most common complication as seen in this case. ${ }^{3}$

The ovarian torsion occurs more frequently during the antenatal period, ${ }^{3,4}$ and this was the scenario in our case. It has been proposed that left ovarian cysts are less prone to have torsion than right ovary, due to the close anatomic relation of the left ovary with the sigmoid colon. ${ }^{5}$ However, in our case it was the left ovary which was torsed.

A systematic review / meta-analysis on outcomes of diagnosed prenatal foetal ovarian cysts concluded that the chances of postnatal surgery were greatly significant in cysts that measure $\geq 40 \mathrm{~mm}$ or if it is a complex cyst. ${ }^{6}$ In this case, the size of the cysts were $\geq 40 \mathrm{~mm}$ with features of intestinal obstruction necessitating a surgical exploration. Antenatal ovarian torsions has been described in previous studies by Akin MA et al., Ozcan HN et al., Ogul et al., and Singal AK et al., the latter case approached laparoscopically., $4,8,9$

Intestinal obstruction by neonatal ovarian cysts is a rare complication. At times it may lead to perforation and peritonitis. The mechanisms implicated in bowel obstruction are either by adhesions or mass effect caused by torsed ovarian cyst. Our case had a diagnostic dilemma, since the left torsed ovary crossed the midline causing a mass effect at the ileocecal junction producing features of intestinal obstruction which could not be delineated by ultrasonography postnatally. Literature search has shown about twenty reported cases of neonatal ovarian torsion complicated by intestinal obstructions, (Nineteen cases compiled in a study by Jeanty $\mathrm{C}$ et al. ${ }^{10}$ and a single case by Akin MA et al. ${ }^{4}$ ).

No specific guidelines have been set for the postnatal management of antenatally detected ovarian cysts and remain controversial. Most cysts have spontaneous resolution, hence a wait and watch policy is advocated. A complex / complicated cyst / symptomatic cyst justifies immediate surgical intervention. Surgical approaches could be either laparotomy or laparoscopy as per the clinical situation. In our case, the former was chosen since there was progressive abdominal distension with features of intestinal obstruction.

\section{CONCLUSIONS}

We highlight an unusual cause of intestinal obstruction due to ovarian torsion in a neonate which presented as antenatally detected abdominal cystic lesion. High index of suspicion to recognise such causes of intestinal obstruction and early surgery could prevent complications like intestinal perforation, peritonitis and neonatal sepsis.

\section{REFERENCES}

1. Trinh TW, Kennedy AM. Foetal ovarian cysts: review of imaging spectrum, differential diagnosis, management and outcome. Radiographics. 2015;35:621-35. DOI: 10.1148/rg.352140073 
2. Chiaramonte C, Piscopa A, Cataliotti F. Ovarian cysts in newborns. Pediatr Surg Int. 2001;17:171-4. DOI: 10.1007/ s003830000471

3. Nussbaum AR, Sanders RC, Hartman JS, Dudgeon DL, Parmley TH. Neonatal ovarian cysts. Sonographic pathologic correlation. Radiology 1988;168:817-21. DOI: 10.1148/radiology.168.3.3043551

4. Akın MA, Akın L, Özbek S, Tireli G, Kavuncuoglu S, Sander S, et al. Fetal-neonatal ovarian cysts: their monitoring and management - retrospective evaluation of 20 cases and review of the literature. J Clin Res Pediatr Endocrinol. 2010; 2:28-33. DOI: $10.4274 /$ jcrpe. v2i1.28.

5. Currarino G, Rutledge JC. Ovarian torsion and amputation resulting in partially calcified, pedunculated cystic mass. Pediatr Radiol. 1989;19:395-9. DOI:10.1007/BF02387636

6. Bascietto F, Liberati M, Marrone L, Khalil A, Pagani G, Gustapane S, et al. Outcome of fetal ovarian cysts diagnosed on prenatal ultrasound examination: systematic review and metaanalysis. Ultrasound Obstet Gynecol. 2017; 50: 20-31. DOI:10.1002/uog.16002.

7. Ozcan HN, Balci S, Ekinci S, Gunes A, Oguz B, Ciftci AO et al. Imaging findings of fetal neonatal cysts complicated with ovarian torsion and autoamputation. AJR Am J Roentgenol.2015; 205:185-9. DOI: 10.2214/AJR. 14.13426

8. Ogul H, Havan N, Pirimoglu B, Guvendi B, Kisaoglu A, Kantarci M. Prenatal and postnatal ultrasonographic findings of torsioned ovarian cyst: a case report and brief literature review. Int Surg. 2015;100: 514-7. DOI: 10.9738/INTSURG-D-14-00005.1

9. Singal AK, Vignesh KG, Paul S, Matthai J. Antenatally diagnosed ovarian cyst managed laparoscopically.J Indian Assoc Pediatr Surg. 2008;13:28-9. DOI: 10.4103/0971-9261.42571.

10. Jeanty C, Frayer EA, Page R, Langenburg S. Neonatal ovarian torsion complicated by intestinal obstruction and perforation and review of the literature. J Pediatr Surg. 2010;45:5-9. DOI: 10.1016/j.jpedsurg.2010.02.118. 